INVESTIGACIÓN

\title{
EVALUACIÓN DEL CAPITAL INTELECTUAL BAJO EL MODELO INTELLECTUS: SINERGIA DE CONOCIMIENTOS Y ESTRATEGIAS EN LA UNIVERSIDAD FRANCISCO DE PAULA SANTANDER OCAÑA

\author{
EVALUATION ON INTELLECTUAL CAPITAL UNDER MODEL \\ INTELLECTUS: SYNERGY OF KNOWLEDGE AND STRATEGIES IN \\ THE UNIIVERSITY FRANCISCO DE PAULA SANTANDER OCAÑA
}

\author{
MSc.Wilder Quintero Quintero ${ }^{\mathrm{a}}$, MSc. Marta Milena Peñaranda Peñaranda ${ }^{\mathrm{b}}$, MSc Magda \\ Mildred Rodriguez Castilla ${ }^{c}$
${ }^{a}$ Universidad Francisco de Paula Santander Ocaña, grupo de investigación GIDSE Calle 7 No. 9-40, Ocaña, Colombia, wquinteroq@ufpso.edu.co
${ }^{\mathrm{b}}$ Universidad Francisco de Paula Santander Ocaña, grupo de investigación GIDSE Calle 11 No.35-150, Ocaña, Colombia, mmpenarandap@ufpso.edu.co
${ }^{\mathrm{c}}$ Universidad Francisco de Paula Santander Ocaña, grupo de investigación GIDSE Calle 8 No.29A-141, Ocaña, Colombia, mmrodriguezc@ufpso.edu.co

\begin{abstract}
Resumen: El Capital Intelectual, pretende dimensionar el conjunto de activos intangibles con que cuentan las organizaciones, de ahí que este estudio centra su atención en la evaluación del capital intelectual en la Universidad Francisco de Paula Santander seccional Ocaña, con un enfoque cuantitativo no experimental de tipo transversal descriptivo, donde a partir del diseño de medición permitió diagnosticar cada uno los elementos que componen el capital intelectual como son: capital humano, capital estructural y capital relacional.

Los resultados de la revisión teórica y la consulta a expertos permitió determinar el modelo Intellectus como el referente teórico para esta investigación. Del análisis estadístico realizado se pudo determinar la necesidad de capacitaciones para el fortalecimiento del capital humano así como del diseño de estrategias que permitan un mayor crecimiento institucional de cara al desarrollo de su capital estructural y sostenibilidad de su capital relacional.
\end{abstract}

Palabras clave: Conocimiento, Capital, Experiencia, Intelectual, Intellectus.

\begin{abstract}
The intellectual capital tries to size the set of intangible assets which count the organizations; from there this study focuses its attention on the evaluation of the intellectual capital in the University Francisco de Paula Santander sectional Ocaña, with a non-transversal
\end{abstract}


quantitative focus, where starting from the measurement design allowed to diagnose every one of the elements that compose the intellectual capital such as: Human capital, structural capital and relational capital.

The results of the theoretical re revision and consult to experts allowed to determine the model Intellectus as a theoretical reference for this investigation. From the statistical analysis realized could be determined the necessity of trainings for the strengthening of the human capital so the design of strategies that permit a higher institutional growth that faces the development of the structural capital and sustainability of the relational capital.

Keywords: Knowledge, capital, experience, intellectual, Intellectus.

\section{INTRODUCCIÓN}

Históricamente la humanidad ha evolucionado sufriendo cambios significativos en cuanto a la generación de riqueza y poder; inicialmente la creación de riqueza estaba centrada en la posesión de tierra, posterior a esto, surgen las manufacturas, las cuales desplazan a la agricultura como fuente principal de riquezas, y finalmente en el siglo $\mathrm{XX}$ se crea el interés de estudiar el capital intelectual en las organizaciones puesto que actualmente los factores de producción (tierra, trabajo y capital) se complementan con el conocimiento, la experiencia, la innovación y la tecnología lo cual implica generar mayores rendimientos económicos para las organizaciones.

El estudio del capital intelectual surge como una necesidad por parte de las empresas, quienes descubrieron que el valor comercial de las organizaciones era superior en el mercado al registrado en los estados financieros, producto de esto la empresa sueca Seguros Skandia publicó el primer informe sobre capital intelectual, el cual se constituyó como información complementaria a los estados financieros de dicha compañía.

En este sentido (Kamukama, 2013), establece que "las organizaciones se encuentran en un continuo ambiente de aprendizaje, ya que el entorno socioeconómico en el que se desenvuelven les exige generar estrategias para sobrevivir ante la competencia existente. Surgiendo así la necesidad de proporcionar un marco coherente para integrar el conocimiento conceptual y empírico para que coincida con dicha volatilidad". De igual manera (Bueno, Salmador, Merino, 2008), afirma que los recursos intangibles de las empresas son su principal fuente de ventaja competitiva sostenible, específicamente se habla de su capital intelectual, el cual genera valor e incrementa el desempeño de éstas.

De igual manera para (López, 2012), muchos investigadores se han dedicado a profundizar el rol del conocimiento, del también denominado capital intelectual, en las empresas y ellas incluyen también las dedicadas al mundo educativo. Para ellas el capital intelectual, el conocimiento, es el rasgo fundamental de organizaciones que reivindican los activos inmateriales como verdadera e indiscutible ventaja competitiva.

De acuerdo a lo planteado anteriormente, se considera el capital intelectual como una sinergia de conocimientos y experiencias del Recurso Humano en las organizaciones, por lo tanto el presente estudio tiene por objeto evaluar el capital intelectual bajo el modelo intellectus en la Universidad Francisco de 
Paula Santander Ocaña, teniendo en cuenta que es el más apropiado para medir dichos activos intangibles en la institución de educación superior a partir de tres dimensiones como son Capital Humano, el Capital Estructural y Capital Relacional.

En concordancia con lo planteado anteriormente, el presente estudio pretende profundizar en el conocimiento de las situaciones, costumbres y actitudes predominantes mediante la descripción exacta de las actividades, objetos, procesos, procedimientos y personas que intervienen en la valoración del capital intelectual en la Universidad Francisco de Paula Santander Ocaña, para la cual se utilizó el tipo de investigación cuantitativa, de tipo de transversal descriptiva.

\section{METODOLOGÍA}

Según las características de la investigación, el diseño de investigación es de tipo cuantitativo, sustentado en el hecho de que la realidad es objetiva, singular y separada del investigador, según (Ruiz, 2012), la investigación cuantitativa busca conocer los hechos reales tal y como se dan objetivamente; y de tipo no experimental de tipo transeccional-descriptiva, donde se hace referencia al proceso de indagación en el que se recogen datos sin la manipulación de variables, buscando a través de ella conocer las dimensiones del capital intelectual utilizando el modelo Intellectus, en la Universidad Francisco de Paula Santander Ocaña.

La población objeto de estudio está conformada por 670 empleados (Administrativos y Docentes) y 5.077 estudiantes matriculados en los distintos programas académicos de la Institución. Teniendo en cuenta que la población objeto de estudio es muy amplio se hace necesario aplicar la formula estadística para poblaciones finitas como se describe a continuación:

$$
\boldsymbol{n}=\frac{\boldsymbol{Z}^{\mathbf{2}} * \boldsymbol{N} * \boldsymbol{p} * \boldsymbol{q}}{\boldsymbol{e}^{\mathbf{2}} *(\boldsymbol{N}-\mathbf{1})+\mathbf{Z}^{\mathbf{2}} * \boldsymbol{p} * \boldsymbol{q}}
$$

La muestra se establece al reemplazar en la formula estadística para poblaciones finitas descrita anteriormente, para la cual se determinó realizar 244 encuestas a empleados (Administrativos y Docentes) y 357 estudiantes de la Universidad Francisco de Paula Santander Ocaña.

En cuanto a la recolección de información, se utilizó como instrumento la encuesta, cuya estructura se divide en tres (3) dimensiones que corresponden a las tres variables que hacen parte del Capital Intelectual (Capital Humano, Capital Estructural y Capital Relacional), a la cual se incorporó la escala Likert, como se aprecia a continuación:

Tabla 1. Escala de valuación de indicadores

\begin{tabular}{|c|c|c|}
\hline Nivel & $\begin{array}{l}\text { Valor } \\
\text { Numérico }\end{array}$ & $\begin{array}{l}\text { Valor } \\
\text { Nominal }\end{array}$ \\
\hline Siempre & 5 & $\begin{array}{l}\text { Muy } \\
\text { Importante }\end{array}$ \\
\hline Casi siempre & 4 & Importante \\
\hline Algunas veces & 3 & Indiferente \\
\hline Ocasionalmente & 2 & $\begin{array}{l}\text { Poco } \\
\text { importante }\end{array}$ \\
\hline
\end{tabular}




$\begin{array}{lll}\text { Nunca } & 1 & \text { Sin } \\ & & \text { importancia }\end{array}$

En este sentido, la muestra seleccionada (Administrativos, docentes y Estudiantes), responden el cuestionario donde pueden seleccionar en cinco (5) opciones de respuesta que corresponden a: Nunca, Ocasionalmente, Algunas Veces, Casi Siempre, Siempre. Dicho cuestionario se constituye por diez y nueve (19) preguntas para docentes $y$ doce preguntas para Administrativos y Estudiantes, las cuales permiten evaluar el capital intelectual en la Universidad Francisco de Paula Santander Ocaña en sus tres dimensiones: Capital Humano, Capital Estructural y Capital Relacional).

Finalmente, con la información suministrada por el instrumento antes mencionado, se realiza un diagnóstico de los sistemas y tecnologías de la información utilizados en la Institución, el capital intelectual y la gestión del conocimiento, y posteriormente, se realiza un análisis de varianza (ANOVA), que permite contrastar la significación estadística de la diferencia de medias entre grupos (Pérez, 2005). Cabe mencionar que los anteriores análisis estadísticos se llevarán a cabo con el software estadístico SPSS y el empleo de Microsoft Excel, para el manejo de la base de datos obtenida.

\section{RESULTADOS}

Concluido el proceso de recolección y tabulación de los datos obtenidos de las encuestas aplicadas 244 empleados (Administrativos y Docentes) y 357 estudiantes de la Universidad Francisco de Paula Santander Ocaña, se procede a realizar el respectivo análisis a partir de las características del capital intelectual en la institución mediante le modelo intellectus, en sus tres dimensiones que son Capital Humano, Capital Estructural y Capital Relacional.

3.1. Dimensión del Capital Humano. En el desarrollo de la investigación en cuanto al Capital Humano de la Universidad Francisco de Paula Santander Ocaña, lo constituyen los conocimientos de cada una de las personas que laboran en la institución, adquiridos a partir de la formación académica y la experiencia que la vida laboral entrega. Dentro de la UFPSO, la fuerza laboral está integrada por los docentes y administrativos, los primeros encargados del cumplimiento de la función misional (academia, investigación y proyección social) de la institución y los segundos los encargados de dar apoyo y soporte a la gestión misional, mediante el desempeño de funciones administrativas.

El cuerpo docente de la UFPS Ocaña está integrado en la actualidad por treinta (30) docentes de carrera, veintinueve (29) con dedicación de tiempo completo y uno (1) con dedicación de medio tiempo; y trescientos (300) docentes de cátedra que prestan su servicio a los diferentes programas académicos, a nivel técnico, tecnológico, profesional y de postgrado, para un total de 330 profesores vinculados.

Entre tanto, el equipo encargado de la función administrativa lo integran 28 administrativos de Planta con más de quince años de permanencia en su cargo, vinculados por concurso de méritos, 146 contratados a término fijo, por lo general inferior a un (1) año, 18 aprendices SENA, 31 auxiliares de servicios generales y 117 personas contratadas mediante orden de prestación de Servicios, para un total de 340 trabajadores contratados. 
El capital Humano Docente de la institución, están distribuidos por facultades y departamentos académicos, los cuales cuentan con nivel de escolaridad así: Ciento veintisiete (127) con pregrado, ciento sesenta y dos (162) con especialización, treinta y ocho (38) con maestría, y Tres (3) docentes con doctorado. En este sentido la universidad capacita a sus docentes a través de los departamentos académicos quieres ofrecen cursos de actualización, así como también a través del plan de capacitación docente ofrece la posibilidad a sus docentes de realizar posgrados que están direccionados especialmente a maestrías y doctorados.

En cuanto a la percepción del capital humano docente de la institución, producto de la recolección y análisis de información, se hacen las siguientes consideraciones: En cuanto a los aspectos académicos en los programas de la institución se realiza la planeación y organización de material para desarrollar los contenidos propios de cada curso o asignatura, de igual manera utilizan con frecuencia las bases de datos que posee la institución.

En cuento a los aspectos de la utilización de las Tecnologías de la Información y Comunicación, los docentes en un $64 \%$ participan en las capacitaciones que la institución realiza, lo cual garantiza el buen uso de los recursos bibliográficos y digitales de la Institución para el desarrollo de actividades en el aula de clases y extra clase; de igual manera los docentes en un $71 \%$ utilizan $\quad 0$ implementan diversas metodologías como foros, discusiones, debates, conversatorios, entre otros, buscando garantizar el aprendizaje de los estudiantes.

De igual manera los docentes siempre utilizan las redes sociales en un $39 \%$ y casi siempre en un $28 \%$ como herramienta que complementa el desarrollo de los contenidos programáticos en el aula de clase con herramientas tecnológicas como la plataforma moodle que permite compartir información a parte de profundizar en los cursos, además de la generación de debate frente a diversas temáticas.

En este mismo sentido, en aspectos de investigación y proyección social, el $18 \%$ de los docentes fomentan la investigación y extensión, mientras que el $37 \%$ casi nunca fomentan de la investigación y la proyección social como pilares fundamentales para el crecimiento y desarrollo académico. También consideran que la investigación es desarrollada principalmente por docentes de tiempo completo, y muy baja por los docentes de vinculación por hora cátedra; de igual manera manifiestan que la publicación de carácter científico es escasa.

En aspectos de espacios físicos, los docentes manifiestan que son insuficientes para el desarrollo de las actividades académicas, para lo cual se deben optimizar el uso de aulas, auditorios, laboratorios y salas de cómputo.

En cuanto a la capacitación docente consideran importante y han participado en eventos dirigidos a la capacitación y actualización docente, con un alto poder de convocatoria que le ha permitido reunir a un alto porcentaje de docentes.

Finalmente manifiestan que la cualificación docente y de los procesos es importante para el desarrollo de la academia, es así como el reconocimiento a los procesos estratégicos, misionales y de apoyo es ratificado por las certificaciones en ICONTEC otorgadas a la Universidad. 
En cuento al Capital Humano de los administrativos, estos cuentan con niveles de escolaridad así: cuarenta y cinco (45) administrativos que no poseen ningún tipo de formación académica, diez y ocho (18) aprendices SENA, setenta y cinco (75) con formación de bachiller, veintinueve (29) con formación de técnica, veinte (20) con formación tecnológica, ciento seis (106) con formación profesional, treinta y cinco (35) con formación de especialización, y doce (12) con formación de maestría. En este sentido la institución cuenta en gran proporción con personal administrativo capacitado, contribuyendo con el desarrollo de los procesos administrativos.

En cuanto a la percepción del capital humano de los administrativos de la institución, producto de la recolección y análisis de información, a través de la aplicación de encuestas, se hacen las siguientes consideraciones: en un $72 \%$ los administrativos planean sus actividades y conocen las funciones propias del cargo o dependencia; de igual manera el $77 \%$ del personal administrativo considera que el Plan de Capacitación dirigido a los administrativos, generalmente ampara a quienes hacen parte de la carrera administrativa, poca participación se muestra por parte de los colaboradores vinculados por contrato a término fijo inferior a un año, por orden de prestación de servicios y aprendices SENA, mientras que el personal docente participa con mayor frecuencia en las capacitaciones y programas que mejoran su cualificación y les ofrece mayores beneficios.

En este mismo sentido, en cuanto a la motivación del personal administrativo, la institución establece estrategias e incentivos que contribuyen con la motivación del personal como capacitación y orientación que aumente su conocimiento y destreza para la ejecución de las labores encomendadas de acuerdo con el cargo que ocupan, y finalmente manifiestan que participan en el diseño y socialización de estrategias de mejora continua a través de los sistemas de gestión de la institución.

\subsection{Dimensión del Capital Estructural.}

Está integrado por la gestión del conocimiento reflejada en la UFPSO, como su plan de desarrollo, proyecto Educativo Institucional (PEI), dirección estratégica, estructura organizacional, procesos documentados, infraestructura, Sistemas de calidad, Investigación + Desarrollo + Innovación, Inversión en activos, entre otros aspectos que facilita el flujo de conocimiento e implica una mejora en la eficiencia de la institución.

La Universidad Francisco de Paula Santander Ocaña tiene plenamente definida su misión, visión, Principios Institucionales, Principios filosóficos, Principios pedagógicos, Principios investigativos, Principios axiológicos, Propósitos Institucionales, de igual manera atendiendo a su compromiso de institución de educación superior de carácter oficial, ha definido políticas de operación que orienten el quehacer institucional y permitan el desarrollo sostenido y el alcance de los objetivos institucionales, dentro de las cuales se destacan la política integral, política de riesgos, Política de Información y Comunicación, política de compromiso frente al MECI, Política de responsabilidad social con la comunidad.

En cuanto a la estructura administrativa y académica, se constituye por organismos de dirección como: $\quad$ El Consejo Superior Universitario, El Rector, El Consejo Académico, El Director de la Seccional; organismos de asesoría: El Comité de Dirección, El Comité de Apoyo Académico, El Comité Administrativo, La Secretaría 
General, La Oficina de Planeación, La Oficina de Relaciones Institucionales e Información, La Oficina de Control Interno; y como organismos de gobierno: La Subdirección Académica, La Subdirección Administrativa, Las facultades, Las Jefaturas de Departamento.

La estructura física en la institución permite desarrollar las actividades académicas y administrativas que se lleva a cabo en el campus Universitario que tiene una extensión total de $1.482 .168 \mathrm{~m} 2$, con un área construida de 38.075 m2en las diferentes sedes, así:

Sede el algodonal (Ubicación a $2.8 \mathrm{Km}$ del Perímetro Urbano). En un área de 125 hectáreas se encuentra construido 38.075,00 M2, en 53 aulas, 29 Laboratorios, Granja Experimental, Auditorios, Biblioteca, Hemeroteca, Unidad Deportiva, Área Administrativa, Área Académica, Centros de Estudios, Bienestar Universitario, Cafeterías, Almacén, División de sistemas y Unidades Sanitarias.

Además la institución ha venido implementando equipos de alta tecnología, para lo cual, la universidad cuenta en todas las dependencias con un sistema de Intranet y sistema de internet inalámbrico para la sede del algodonal con capacidad de más de 2000 equipos conectados al mismo tiempo, se han implementado salas de informática, se cuenta con la sistematización de la información bibliográfica (SIB), el Sistema de Información Académica (SIA), el Sistema de Información Financiera (SIF), Sistema Integrado de Gestión MECI - Calidad, con la articulación de las normas ISO 90001:2008, NTCGP1000:2009 y MECI 2005; Sistema de gestión ambiental y salud ocupacional ISO 14001:2004 y OHSAS 18001:2007 con el objeto de propender por el mejoramiento de las condiciones técnicas de los estamentos docentes y administrativos.

En la sede de Bellas Artes, se cuenta con un auditorio múltiple que permite realizar actividades como ceremonias de grados, conferencias, seminarios y demás eventos complementarios y las aulas de música.

Sede La Primavera (Ubicada en la ciudad). Esta sede está situada en la Avenida Francisco Fernández de Contreras de la ciudad de Ocaña, con un área construida de $4.655 \mathrm{~m} 2$, el espacio físico de este edificio permite contar con alternativas de ampliación y adecuación según las necesidades de la universidad.

También hace parte del capital estructural la autoevaluación institucional para la medición, análisis y mejora continua, la cual es concebida como un proceso de reflexión permanente y de obtención de información oportuna y eficaz para la toma de decisiones en pro del mejoramiento continuo y la obtención de la calidad esperada de los programas académicos y la Institución en su conjunto.

Otro aspecto importante que contribuye con el capital estructural de la institución es la Investigación + Desarrollo + Innovación, los cuales están conformados por el Centro de Desarrollo e Innovación Tecnológica (CEDIT), encargado de generar conocimiento, desarrollo e innovación tecnológica en las diferentes áreas de la ciencia, especialmente en las relacionadas con las Tecnologías de la Información y las Comunicaciones (TIC); el Centro de Investigación para Desarrollo Regional (CIDER), encargado de diseñar y desarrollar investigación de índole local, regional, nacional e internacional; el Centro de Investigaciones del Sector Solidario 
(CEISS), el cual promueve, organiza y coordina el proceso de formación, capacitación e investigación en el Sector Solidario.

De otra parte en la UFPSO se desarrolla Investigación, Innovación y Extensión, a través de la división de investigación y extensión (DIE), cuyo objetivo principal es el fomento, organización, dirección y supervisión de las actividades de investigación y extensión que realizan las Facultades y los Departamentos de la institución.

\subsection{Dimensión del Capital Relacional.} Está directamente relacionado con la satisfacción de sus clientes internos, externos y públicos de interés, manteniendo una relación directa con sus colaboradores, estudiantes y egresados principalmente, con programas de extensión y proyección social orientados al desarrollo en comunidades de la región y su área de influencia.

En este sentido, hacen parte del capital relacional de la institución los clientes internos conformados por todas las dependencias o áreas de la UFPSO, a las cuales se realiza una evaluación de satisfacción del cliente interno, reflejándose una calificación favorable, superior al $70 \%$ de satisfacción.

En cuento a los clientes externos está conformado por los estudiantes de los programas académicos de la institución, procedentes de 29 de los 32 departamentos de la geografía nacional, que ven en ella una oportunidad para adelantar sus estudios superiores por ser una universidad pública, dichos estudiantes ascienden a 5.077 actualmente, donde el $87,3 \%$ de los estudiantes se encuentran matriculados en programas profesionales presenciales de los cuales $\quad 0,48 \%$ corresponden a especialización, el $9,1 \%$ en programas técnicos y tecnológicos presenciales y el $3,5 \%$ de los estudiantes están matriculados en programas a distancia.

Otro aspecto importante en el capital relacional de la UFPSO, son sus egresados los cuales ascienden a 6.694 profesionales en sus diferentes modalidades, los cuales general el impacto de la institución en el sector externo, razón por la cual existe una relación muy cercana con dichos egresados, los cuales son vinculados con la Institución, realizando seguimiento permanente a la labor que desarrollan estos profesionales en el sector empresarial, para determinar fortalezas y debilidades en la formación de profesionales idóneos, contribuyendo de esta manera con los procesos de autoevaluación y mejoramiento continuo de la calidad de los programas que ofrece la Universidad Francisco de Paula Santander Ocaña.

\section{CONCLUSIONES.}

La medición del capital intelectual en la Universidad Francisco de Paula Santander Ocaña, se desarrolló mediante la aplicación del modelo Intellectus, donde se pudo evidenciar que dicha institución de educación superior cuenta con criterios y políticas institucionales en materia de adquisición y actualización de recursos informáticos y de comunicación, configurando los Sistemas de Información como una herramienta tecnológica que permite la interrelación de los procesos académicos, de investigación y extensión mediante herramientas que se han diseñado a propósito de las necesidades de desarrollo Académico de la Institución.

Asimismo, en la búsqueda por la simplificación y mejoramiento de los servicios administrativos, se han dirigido esfuerzos para disponer de una organización moderna con servicios eficientes y oportunos. Por esto el Proceso de Sistemas de Información, Telecomunicaciones y 
Tecnologías, es el encargado de velar por el buen funcionamiento de los recursos informáticos y de comunicación para apoyar el desarrollo de las distintas actividades académicas y Administrativas de la Universidad.

Al considerar al capital humano e intelectual como un intangible fundamental para el funcionamiento de la Institución, la UFPSO, propende por la oferta permanente de programas de capacitación y actualización dirigido a docentes y administrativos de todos los niveles jerárquicos de su estructura orgánica, logrando implementar en su interior una cultura de autoevaluación y mejoramiento continuo que garantice la alta calidad de sus procesos, la satisfacción de los clientes y el desempeño de la gestión de la institucional promulgado en su misión.

Los distintos modelos de medición del capital intelectual presentan aspectos que orientan el proceso al interior de la organización, cuya elección depende del criterio de quienes la integran y de los objetivos que se propongan con esta medición. En este sentido se definió el modelo Intellectus para realizar la medición del capital intelectual en la Universidad Francisco de Paula Santander Ocaña, teniendo en cuenta que es el modelo que muestra mayor afinidad con las características de la institución a través de las dimensiones de Capital Humano, Capital Estructural y Capital Relacional.

\section{FINANCIACIÓN}

El proyecto. Medición del Capital intelectual en la Universidad Francisco de Paula Santander Ocaña, fue realizado gracias a la financiación realizada por dicha institución.

\section{REFERENCIAS BIBLIOGRÁFICAS}

Bueno, E., Salmador, M.P. y Merino, C. (2008). Génesis, concepto y desarrollo del capital intelectual en la economía del conocimiento: Una reflexión sobre el Modelo Intellectus y sus aplicaciones, Red de Revistas Científicas de América Latina, el Caribe, España y Portugal, 26 (2), 43-63.

Kamukama, N. (2013). Intellectual capital: company's invisible source of competitive advantage, Competitiveness Review: An International Business Journal, 23 (3), 260.

López, M. (2012). Gerencia: capital intelectual y sus competencias en instituciones educativas. Observatorio Laboral, 120.

López, M. (2012). Gerencia: capital intelectual y sus competencias en instituciones educativas. Observatorio Laboral, 120.

Ruiz, J. I. (2012). Teoría y Práctica de la Teoría Cuantitativa. Bilbao: Publicaciones de la Universidad de Deusto. 